\title{
Accounting Conservatism as a Social Norm
}

Jivas Chakravarthy and Timothy Shields

- We create a setting which pairs a Sender and Receiver that is characterized by

i. information asymmetry;

ii. measurement uncertainty; and

iii. misaligned incentives that motivate senders to report aggressively

- The first two characteristics create a verification problem that heightens attention on the ex post reporting error the only information that Receivers observe

- The third characteristic makes the Receiver wary of the Senders' intentions, as reporting errors of greater value indicate a greater likelihood of exploitative intent

- Experiment: 6 Senders and 6 Receivers interact in S-R pairs for 10 periods

- End of each period: Rs rank each Sender, determining the next period's pairings $\rightarrow$ revealed preferences
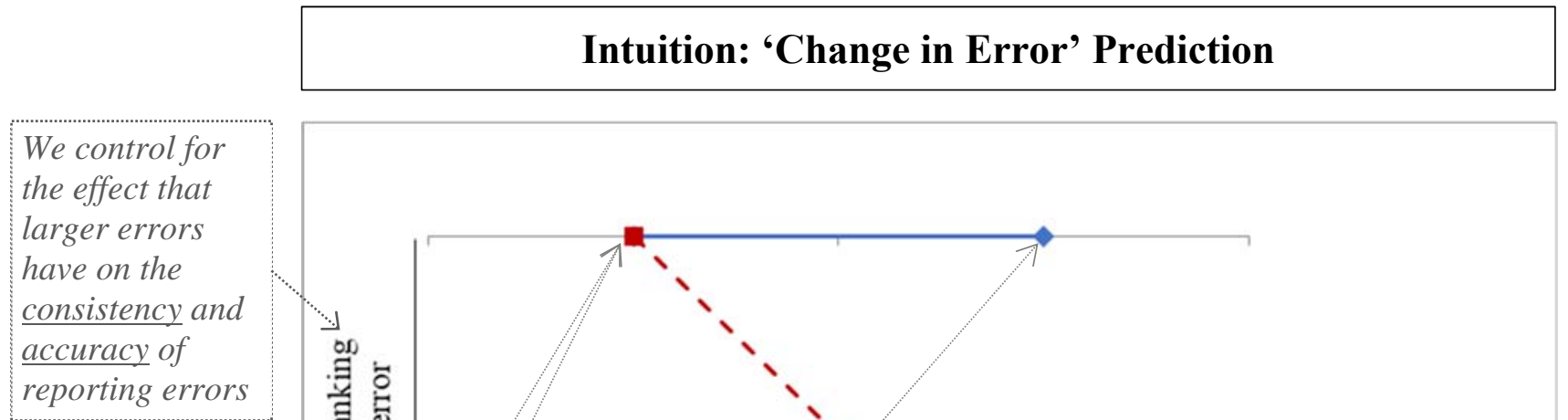

We control for the effect that larger errors have on the consistency and accuracy of reporting errors

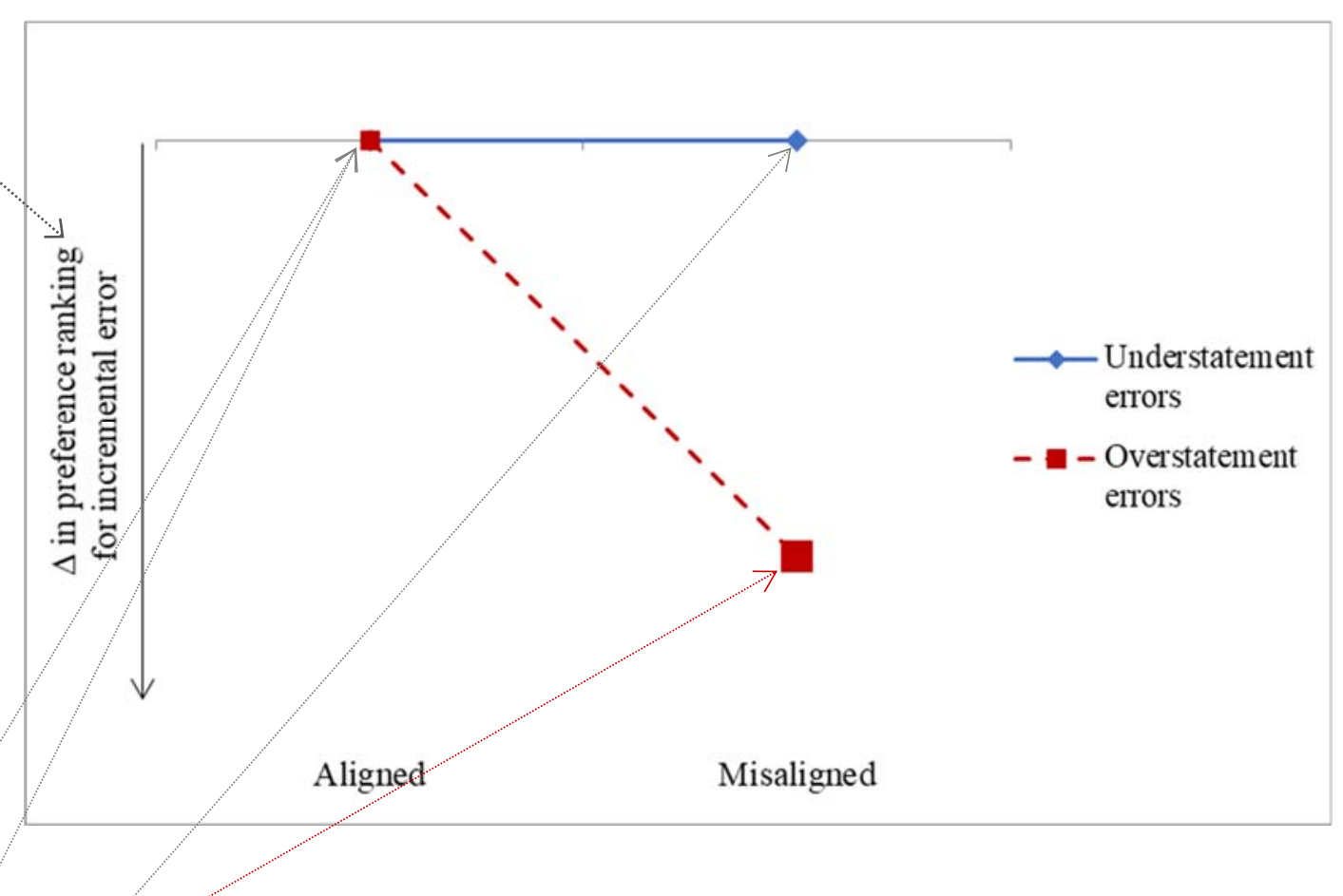

In the first three outcomes there are no factors outside of consistency and accuracy that lead Receivers to disprefer larger magnitude errors - but when Senders are motivated to report aggressively, we predict (and find) that an incremental overstatement error is associated with a significant decline in their average rank

In sum, we find that Receivers have asymmetric preferences over ex post reporting errors that are consistent with a normative opposition to aggressive reporting

- The revealed preferences are inconsistent with self-interested behavior and cannot be explained by loss aversion

- Caveat: our evidence is both indirect and does not satisfy all conditions for a social norm to exist (e.g., Bicchieri 2006)

- However, it opens the possibility that conservatism emerged as a social norm - an informal bonding cost, borne by the sender - that arises endogenously through repeat interaction and that minimizes 'contracting' losses arising from information asymmetry 